\title{
Avaliação da padronização das sondas periodontais do tipo Williams e OMS de diferentes marcas
}

Evaluation of standardization of Williams and WHO periodontal probes of different brands

Evaluación de la estandarización de sondas periodontales Williams y OMS de diferentes marcas

\author{
Cristiane TONUS $^{1}$ \\ Natália SOBRAL ${ }^{1}$ \\ Douglas FERLA ${ }^{1}$ \\ Daniel GALAFASSI ${ }^{2}$ \\ Juliane Pereira BUTZE ${ }^{3}$
}

${ }^{1}$ Graduado(a) do Curso de Odontologia do Centro Universitário da Serra Gaúcha - FSG, Caxias do Sul, Brasil

${ }^{2}$ Doutor em Dentística Restauradora pela USP, Docente do Curso de Graduação em Odontologia, Centro Universitário da Serra Gaúcha (FSG) 95020 - 472 Caxias do Sul - RS, Brasil

${ }^{3}$ Doutora em Periodontia pela UFRGS, Docente do Curso de Graduação em Odontologia do Centro Universitário da Serra Gaúcha (FSG) 95020 - 472 Caxias do Sul - RS, Brasil

\section{Resumo}

Objetivos: O estabelecimento do diagnóstico clínico periodontal tem como base a avaliação de parâmetros clínicos por meio da sondagem periodontal, sendo através desses resultados que o Cirurgião Dentista fará a melhor escolha de tratamento. Porém, possíveis erros encontrados na padronização das sondas periodontais podem levar a um plano de tratamento inadequado e/ou ineficiente. O presente estudo teve como objetivo avaliar a padronização de seis sondas periodontais do tipo Williams e seis sondas do tipo OMS de diferentes marcas comerciais. Materiais e Métodos: Com o auxílio de um paquímetro digital e de uma balança digital de alta precisão, foi avaliado o peso da sonda, o diâmetro do cabo, o diâmetro da ponta ativa/esfera, e distância das marcações. Além disso, foi feito uma comparação dos preços de cada sonda, para que fosse possível se avaliar o custo/benefício de cada sonda periodontal. Resultados: Os resultados demonstraram que as sondas das seis marcas comerciais avaliadas, tanto das sondas do tipo OMS quanto do tipo Williams apresentaram algum tipo de distorção em relação às medidas consideradas ideais pela OMS (Organização Mundial da Saúde). Conclusão: As sondas da marca Hu-Friedy, tanto do tipo OMS quanto Williams, apresentaram melhores marcações milimetradas entre todas as marcas avaliadas.

Descritores: Periodontia; Instrumentos; Sondagem Periodontal.

\section{Abstract}

Objectives: The establishment of the periodontal clinical diagnosis is based on the evaluation of clinical parameters through periodontal probing, and through these results the dentist will make the best treatment choice. However, possible errors found in the standardization of periodontal probes may lead to an inadequate and/or inefficient treatment plan. The aim of the present study was to evaluate the standardization of six Williams-type periodontal probes and six WHO-type probes of different trademarks. Materials and Methods: With the aid of a digital caliper and a high precision digital scale, the weight of the probe, the cable diameter, the active tip/sphere diameter, and the distance of the markings were evaluated. In addition, a price comparison of each probe was made, so that the cost/benefit of each periodontal probe could be evaluated. Results: The results showed that the probes of the six evaluated trademarks of both the WHO and Williams probes presented some kind of distortion in relation to the measures considered ideal by the WHO (World Health Organization). Conclusion: The Hu-Friedy probes, both WHO and Williams, had better millimeter markings among all evaluated brands.

Descriptors: Periodontics; Instruments; Periodontal Survey.

\section{Resumen}

Objetivos: El establecimiento del diagnóstico clínico periodontal se basa en la evaluación de parámetros clínicos mediante sondaje periodontal, y es a través de estos resultados que el odontólogo tomará la mejor opción de tratamiento. Sin embargo, los posibles errores encontrados en la estandarización de las sondas periodontales pueden dar lugar a un plan de tratamiento inadecuado y/o ineficaz. El presente estudio tuvo como objetivo evaluar la estandarización de seis sondas periodontales tipo Williams y seis sondas tipo OMS de diferentes marcas comerciales. Materiales y Métodos: Con la ayuda de un calibre digital y una balanza digital de alta precisión, se evaluó el peso de la sonda, el diámetro del cable, el diámetro de la punta / esfera activa y la distancia de las marcas. Además, se realizó una comparación de los precios de cada sonda, de manera que se pudo evaluar el costo/beneficio de cada sonda periodontal. Resultados: Los resultados mostraron que las sondas de las seis marcas comerciales evaluadas, tanto del tipo OMS como del tipo Williams, presentaron algún tipo de distorsión en relación a las medidas consideradas ideales por la OMS (Organización Mundial de la Salud). Conclusión: Las sondas de la marca Hu-Friedy, tanto del tipo OMS como Williams, presentaron mejores marcas en milímetros entre todas las marcas evaluadas.

Descriptores: Periodoncia; Instrumentos; Sondeo Periodontal.

INTRODUÇÃO

Doença periodontal é um processo inflamatório, causada por biofilme bacteriano, que acomete 0 periodonto de proteção (gengiva) e o periodonto de sustentação (cemento, ligamento periodontal e osso) dos dentes ${ }^{1}$. A doença consiste em uma migração apical do epitélio juncional, com perda de estrutura óssea, resultando na formação de bolsas periodontais e consequentemente, retração gengival, mobilidade e perda dentária em casos mais graves ${ }^{2}$. O estabelecimento de um diagnóstico é muito importante para o sucesso do tratamento periodontal, pois é através dele que o Cirurgião Dentista irá determinar o tratamento mais adequado para o caso, identificando se há ou não presença da doença periodontal, a sua extensão e gravidade, para elaborar um plano de tratamento de acordo com a necessidade de cada paciente ${ }^{3}$. O instrumento mais utilizado para diagnóstico clínico periodontal é a sonda periodontal milimetrada, através dessa sonda é possível avaliar alguns parâmetros clínicos, como: profundidade de sondagem (PS), perda de inserção $(\mathrm{PI})$ e sangramento à sondagem $(\mathrm{SS})^{4}$. Porém, alguns fatores podem interferir nos valores obtidos por meio da sondagem periodontal. Tais fatores, normalmente, estão 
relacionados com o diâmetro da sonda, a pressão aplicada pelo profissional ao sondar 0 paciente, a angulação incorreta da sonda no momento da sondagem, o grau de inflamação dos tecidos periodontais, presença de cálculos, restaurações mal adaptadas e defeitos anatômicos do dente ${ }^{4,5,6,7,8}$. Entre eles, também podemos afirmar que algumas características das sondas periodontais milimetradas como o diâmetro da ponta ativa, as marcações milimetradas e o peso da mesma, podem ter suas limitações, interferindo nos valores da sondagem. Esses erros, inerentes à sondagem, podem levar o profissional a realizar um diagnóstico incorreto e, consequentemente, a um tratamento inadequado ${ }^{9}$.

A sonda periodontal com características ideais recomendadas pela Organização Mundial de Saúde (OMS) segue alguns requisitos dimensionais, para que não ocorram erros na sondagem derivadas do próprio instrumento, são eles: peso máximo de $4,5 \mathrm{~g}$; mostrar marcações corretas de milimetragem, ter um cabo com diâmetro de $3,5 \mathrm{~mm}$ e, para as sondas do tipo OMS, a esfera contida no fim da ponta ativa da sonda deve ter $0,5 \mathrm{~mm}$ de diâmetro ${ }^{10}$. As avaliações e estudos das sondas milimetradas periodontais ainda são insuficientes e com isso, o profissional da área da Odontologia deve tentar minimizar os erros dependentes do examinador, para se chegar num diagnóstico periodontal mais preciso possível ${ }^{10,11}$.

Considerando a importância de uma correta sondagem periodontal para um diagnóstico e tratamento adequado, este trabalho tem como objetivo avaliar a precisão do instrumento para verificar se o mesmo possui a padronização correta pela OMS, permitindo uma melhor mensuração das condições periodontais. MATERIAL E MÉTODO

Para a análise, foram utilizadas seis sondas periodontais do tipo William e seis sondas periodontais do tipo OMS de seis marcas comerciais diferentes, sendo elas $\mathrm{Hu}$ Friedy/USA ${ }^{\circledR}$ (Hu-Friedy MFG. Co., Chicago-IL), Fava Metalúrgica ${ }^{\circledR}$ (Metalúrgica Fava Indústria e Comércio, São Paulo-SP), Millenniun/Golgran ${ }^{\circledR}$ (Golgran Indústria e Comércio de Instrumental Odontológico Ltda., São Paulo-SP), Alprime Dental $^{\circledR} \quad$ (Prime Dental Products Pvt.Ltda.,Bhiwandi-Indía), Quinelato Qualidade ${ }^{\circledR}$ (Schobell Industrial Ltda., Rio Claro-SP) e Trinity Odontologia $^{\circledR}$ (Trinity Indústria e Comércio Ltda., São Paulo-SP). Todas as sondas eram novas, sem uso, sem sinais de danos e sem passar pelo processo de autoclave. Foi avaliado o peso da sonda, o diâmetro do cabo, o diâmetro da ponta ativa/esfera, e distância das marcações.

Todas as sondas foram avaliadas por um único examinador. Para a análise do peso das sondas em gramas, utilizou-se uma balança de precisão digital da marca Casita ${ }^{\circledR}$ (Imporiente Com. Ext. Ltda., China), calibrada em gramas, numa superfície firme e plana e em ambiente sem corrente de ar para que não interferisse nas medidas. Cada sonda foi depositada delicadamente sobre a balança e, somente quando os números se tornavam estáveis, era anotado seu peso. Este procedimento foi realizado duas vezes consecutivas a fim de se obter a melhor confirmação possível.

Para avaliar o diâmetro do cabo, foi usado um paquímetro digital de quatro dígitos da marca Digimess ${ }^{\circledR}$ (Digimess Instrumentos de Precisão Ltda., São Paulo-SP) (modelo 100.174BL). Para esta análise, a sonda foi segurada com as mãos pelo examinador, o mesmo sempre permanecia com os braços apoiados em superfície plana e firme, para evitar deslizes e erro de mensuração. Foram utilizadas as garras do paquímetro para medição, no meio do cabo de cada sonda, usando a trava do paquímetro para cada medição. Cabe ressaltar que a cada mensuração, o paquímetro foi recolocado na posição inicial (zero), a fim de se evitar uma leitura viciada. Este procedimento também foi realizado duas vezes consecutivas para melhor confirmação. Este mesmo procedimento foi feito para mensurar o diâmetro da ponta ativa.

Para a mensuração das marcações milimetradas, também foi usado o paquímetro digital. As sondas tinham marcações no $1^{\circ}, 2^{\circ}$, $3^{\circ}, 5^{\circ}, 7^{\circ}$, $8^{\circ}$, 9으 e 10ำ milímetros (sonda Williams), entretanto, foram verificadas as medidas de $0-^{\circ}$, de $0-5^{\circ}, 0-7^{\circ}$ e $0-10^{\circ}$ milímetros. Para as sondas do tipo OMS, foram verificadas as medidas da ponta da esfera até cada marcação $(0-3,5 \mathrm{~mm}, 0-5,5 \mathrm{~mm}, 0-8,5 \mathrm{~mm}$ e $0-11,5 \mathrm{~mm})$. Todas as medidas foram realizadas pelo mesmo avaliador, obedecendo assim os mesmos critérios anteriormente descritos.

Foi realizada também, uma comparação dos preços de cada sonda, em quatro estabelecimentos de produtos odontológicos diferentes (duas lojas virtuais e duas lojas físicas) para que fosse possível se avaliar o custo/benefício de cada sonda periodontal.

RESULTADOS

A Tabela 1 expressa o peso das sondas, a medida do diâmetro do cabo e da ponta ativa das sondas do tipo Williams das seis marcas comerciais. A sonda mais leve foi da marca 
Allprime (09g), seguida da Trinity (14g), Quinelato e Millenium com o mesmo peso $(15 \mathrm{~g})$, Fava (18g) e, por fim, a da Hu-Friedy (20g). Em relação ao diâmetro do cabo, a sonda da marca Fava apresentou o menor diâmetro $(4,96 \mathrm{~mm})$ e a da marca Hu-Friedy apresentou o maior $(9,45 \mathrm{~mm})$, as demais sondas apresentaram diâmetros muito semelhantes. Em relação ao diâmetro da ponta ativa, o valor mais discrepante foi da marca Fava, com $0,77 \mathrm{~mm}$, as demais marcas apresentaram diâmetros parecidos.

Tabela 1. Tabela de resultados do peso das seis marcas comerciais diferentes das sondas periodontais do tipo Williams, diâmetro do cabo e diâmetro da ponta ativa das mesmas

\begin{tabular}{l|l|l|l}
\hline $\begin{array}{l}\text { MARCA } \\
\text { COMERCIAL }\end{array}$ & $\begin{array}{l}\text { PESO DA DIAMETRO DO } \\
\text { SONDA }\end{array}$ & $\begin{array}{l}\text { DIAMETRO DA } \\
\text { CABO }\end{array}$ \\
\hline Hu Friedy & $20 \mathrm{~g}$ & $9,45 \mathrm{~mm}$ & $0,50 \mathrm{~mm}$ \\
\hline Fava & $18 \mathrm{~g}$ & $4,96 \mathrm{~mm}$ & $0,77 \mathrm{~mm}$ \\
\hline Allprime & $9 \mathrm{~g}$ & $7,93 \mathrm{~mm}$ & $0,58 \mathrm{~mm}$ \\
\hline Trinity & $14 \mathrm{~g}$ & $7,83 \mathrm{~mm}$ & $0,54 \mathrm{~mm}$ \\
\hline Quinelato & $15 \mathrm{~g}$ & $7,81 \mathrm{~mm}$ & $0,56 \mathrm{~mm}$ \\
\hline Millennium & $15 \mathrm{~g}$ & $7,97 \mathrm{~mm}$ & $0,59 \mathrm{~mm}$ \\
\hline
\end{tabular}

$\mathrm{Na}$ Tabela 2 estão expressos os valores de peso, diâmetro do cabo e da ponta ativa das sondas do tipo OMS. Em relação ao peso, a sonda mais leve foi da marca Allprime $(09 \mathrm{~g})$, seguida da Trinity (11g), Quinelato (14g), Millenium (15g), Fava (16g) e, por fim, da HuFriedy $(21 \mathrm{~g})$. Em relação ao diâmetro do cabo, se percebeu uma grande variação quantos às medidas obtidas. A sonda da marca Trinity apresentou a menor medida $(3,95 \mathrm{~mm})$, seguida da Fava $(4,94 \mathrm{~mm})$, Quinelato $(7,79 \mathrm{~mm})$, Allprime $(7,87 \mathrm{~mm})$, Millenium $(8,06 \mathrm{~mm})$ e $\mathrm{Hu}-$ Friedy $(9,46 \mathrm{~mm})$. A medida do diâmetro da esfera variou, entre as marcas, de $0,51 \mathrm{~mm}(\mathrm{Hu}-$ Friedy) até $0,68 \mathrm{~mm}$ (Quinelato).

Tabela 2. Tabela de resultados do peso das seis marcas comerciais diferentes das sondas do tipo OMS, diâmetro do cabo e diâmetro da esfera das mesmas.

\begin{tabular}{l|l|l|l}
$\begin{array}{l}\text { MARCA } \\
\text { COMERCIAL }\end{array}$ & $\begin{array}{l}\text { PESO DA } \\
\text { SONDA }\end{array}$ & $\begin{array}{l}\text { DIAMETRO DO } \\
\text { CABO }\end{array}$ & $\begin{array}{l}\text { DIAMETRO DA } \\
\text { ESFERA }\end{array}$ \\
\hline Hu Friedy & $21 \mathrm{~g}$ & $9,46 \mathrm{~mm}$ & $0,51 \mathrm{~mm}$ \\
\hline Fava & $16 \mathrm{~g}$ & $4,94 \mathrm{~mm}$ & $0,61 \mathrm{~mm}$ \\
\hline Allprime & $9 \mathrm{~g}$ & $7,87 \mathrm{~mm}$ & $0,60 \mathrm{~mm}$ \\
\hline Trinity & $11 \mathrm{~g}$ & $3,95 \mathrm{~mm}$ & $0,58 \mathrm{~mm}$ \\
\hline Quinelato & $14 \mathrm{~g}$ & $7,79 \mathrm{~mm}$ & $0,68 \mathrm{~mm}$ \\
\hline Millennium & $15 \mathrm{~g}$ & $8,06 \mathrm{~mm}$ & $0,66 \mathrm{~mm}$ \\
\hline
\end{tabular}

Os dados demonstrados na Tabela 3 comparam as marcações milimetradas $(0-1 \stackrel{0}{ }, 0$ $5^{\circ}, 0-7^{\circ}$ e $\left.0-10^{\circ}\right)$ entre as seis diferentes marcas de sondas do tipo Williams. É possível observar que houve grande variação das medidas, sem grande discrepância entre as marcas avaliadas.

$\mathrm{Na}$ Tabela 4 estão demonstradas as medidas das marcações milimetradas das seis diferentes marcas de sondas do tipo OMS (0$3,5 \mathrm{~mm}, 0-5,5 \mathrm{~mm}, 0-8,5 \mathrm{~mm}$ e $0-11,5 \mathrm{~mm})$. É possível verificar que os valores das medidas das marcações milimetradas são muito semelhantes entre as diferentes marcas, no entanto, a sonda da marca Fava foi a única que apresentou medidas muito aquém das preconizadas pela OMS.
Tabela 3. Resultado das mensurações das marcações milimetradas das seis marcas comerciais diferentes das sondas periodontais do tipo Williams com marcações de 0-1, 0-5, 0-7 e 0-10mm

\begin{tabular}{l|l|l|l|l}
$\begin{array}{l}\text { MARCA } \\
\text { COMERCIAL }\end{array}$ & $\mathbf{0 - 1 \mathrm { mm }}$ & $\mathbf{0 - 5 \mathrm { mm }}$ & $\mathbf{0 - 7 \mathrm { mm }}$ & $\mathbf{0 - 1 0 \mathrm { mm }}$ \\
\hline Hu Friedy & $1,10 \mathrm{~mm}$ & $4,93 \mathrm{~mm}$ & $6,98 \mathrm{~mm}$ & $9,99 \mathrm{~mm}$ \\
\hline Fava & $1,07 \mathrm{~mm}$ & $4,7 \mathrm{~mm}$ & $7,4 \mathrm{~mm}$ & $10,09 \mathrm{~mm}$ \\
\hline Allprime & $0,96 \mathrm{~mm}$ & $5,04 \mathrm{~mm}$ & $6,96 \mathrm{~mm}$ & $10,02 \mathrm{~mm}$ \\
\hline Trinity & $0,92 \mathrm{~mm}$ & $4,94 \mathrm{~mm}$ & $6,93 \mathrm{~mm}$ & $9,97 \mathrm{~mm}$ \\
\hline Quinelato & $1,00 \mathrm{~mm}$ & $5,04 \mathrm{~mm}$ & $6,95 \mathrm{~mm}$ & $10,23 \mathrm{~mm}$ \\
\hline Millennium & $1,07 \mathrm{~mm}$ & $4,93 \mathrm{~mm}$ & $7,08 \mathrm{~mm}$ & $10,08 \mathrm{~mm}$ \\
\hline
\end{tabular}

Tabela 4. Resultado das mensurações das marcações milimetradas dasseis marcas comerciais diferentes das sondas periodontais do tipo OMS com marcações de $0-3,5 \mathrm{~mm}, 0-5,5 \mathrm{~mm}, 0-8,5 \mathrm{~mm}$ e $0-11,5 \mathrm{~mm}$

\begin{tabular}{|c|c|c|c|c|c|}
\hline $\begin{array}{l}\text { MARCA } \\
\text { COMERCIAL }\end{array}$ & $\begin{array}{l}\text { PONTA } \\
\text { ATIVA }\end{array}$ & $0-3,5$ & $0-5,5$ & $0-8,5$ & $0-11,5$ \\
\hline Hu Friedy & $1,10 \mathrm{~mm}$ & $4,93 \mathrm{~mm}$ & $6,98 \mathrm{~mm}$ & $8,49 \mathrm{~mm}$ & $11,51 \mathrm{~mm}$ \\
\hline Fava & $1,07 \mathrm{~mm}$ & $4,71 \mathrm{~mm}$ & $7,4 \mathrm{~mm}$ & $7,71 \mathrm{~mm}$ & $10,64 \mathrm{~mm}$ \\
\hline Allprime & $0,96 \mathrm{~mm}$ & $5,04 \mathrm{~mm}$ & $6,96 \mathrm{~mm}$ & $8,59 \mathrm{~mm}$ & $11,53 \mathrm{~mm}$ \\
\hline Trinity & $0,92 \mathrm{~mm}$ & $4,94 \mathrm{~mm}$ & $6,93 \mathrm{~mm}$ & $8,29 \mathrm{~mm}$ & $11,40 \mathrm{~mm}$ \\
\hline Quinelato & $1, \mathrm{OOmm}$ & $5,04 \mathrm{~mm}$ & $6,95 \mathrm{~mm}$ & $8,64 \mathrm{~mm}$ & $11,63 \mathrm{~mm}$ \\
\hline Millennium & $1,07 \mathrm{~mm}$ & $4,93 \mathrm{~mm}$ & $7,08 \mathrm{~mm}$ & $8,49 \mathrm{~mm}$ & $11,47 \mathrm{~mm}$ \\
\hline
\end{tabular}

A Tabela 5 expressa o valor mínimo e máximo (em reais) encontrados nos preços das seis marcas de sonda do tipo Williams avaliadas. Os valores variaram de $R \$ 45,40$ (Millenium) até $\mathrm{R} \$ 165,00$ (Hu-Friedy), sendo a sonda da marca Hu-Friedy a que apresentou maior custo, bem como, maior variação no valor.

Tabela 5. Tabela de valor mínimo e máximo (em reais) encontrados nos preços das seis marcas de sonda do tipo Williams avaliadas.

\begin{tabular}{l|l|l}
\hline MARCA COMERCIAL & Custo Máximo & Custo Mínimo \\
\hline Hu Friedy & $\mathrm{R} \$ 165, \mathrm{OO}$ & $\mathrm{R} \$ 120,00$ \\
\hline Fava & $\mathrm{R} \$ 24,94$ & $\mathrm{R} \$ 24,00$ \\
\hline Allprime & $\mathrm{R} \$ 40,15$ & $\mathrm{R} \$ 32,90$ \\
\hline Trinity & $\mathrm{R} \$ 37,90$ & $\mathrm{R} \$ 37,05$ \\
\hline Quinelato & $\mathrm{R} \$ 53,08$ & $\mathrm{R} \$ 50,43$ \\
\hline Millennium & $\mathrm{R} \$ 46,65$ & $\mathrm{R} \$ 45,40$ \\
\hline
\end{tabular}

$\mathrm{Na}$ Tabela 6 estão expressos os valores mínimos e máximos (em reais) encontrados nos preços das seis marcas de sonda do tipo OMS avaliadas. Para este tipo de sonda, os valores variaram de $R \$ 29,90$ (Allprime) até $R \$ 215,00$ (Hu-Friedy), sendo esta última marca a que apresentou maior custo e maior variação no valor, semelhante à sonda do tipo Williams da mesma marca.

Tabela 6. Tabela de valor mínimo e máximo (em reais) encontrados nos preços das seis marcas de sonda do tipo OMS avaliadas

\begin{tabular}{l|l|l} 
MARCA COMERCIAL & Custo Máximo & Custo Mínimo \\
\hline Hu Friedy & $\mathrm{R} \$ 215,00$ & $\mathrm{R} \$ 165,00$ \\
\hline Fava & $\mathrm{R} \$ 39,62$ & $\mathrm{R} \$ 40,53$ \\
\hline Allprime & $\mathrm{R} \$ 32,90$ & $\mathrm{R} \$ 29,90$ \\
\hline Trinity & $\mathrm{R} \$ 37,75$ & $\mathrm{R} \$ 34,24$ \\
\hline Quinelato & $\mathrm{R} \$ 50,43$ & $\mathrm{R} \$ 48,57$ \\
\hline Millennium & $\mathrm{R} \$ 60,10$ & $\mathrm{R} \$ 46,65$ \\
\hline
\end{tabular}

DISCUSSÃO

A Doença Periodontal é uma infecção crônica produzida por bactérias gram-negativas, em níveis elevados, causada por acúmulo de componentes microbianos do biofilme dental que se acumulam no interior dos tecidos do periodonto $^{12}$. A doença periodontal é universal, - que significa que qualquer pessoa, independentemente do gênero, faixa etária, etnia e fatores sistêmicos, possa vir a desenvolver ${ }^{13}$.

Sabemos que o tratamento periodontal é de suma importância para combater a gengivite e a periodontite, visando restabelecer a saúde do paciente, diminuindo a recorrência da 
doença e, consequente perda dentária ${ }^{14,15,16}$ Com isso, o uso rotineiro da sonda periodontal é indispensável para o Cirurgião Dentista que deseja acompanhar a saúde de seus pacientes. Porém, existem fatores que podem interferir nos valores obtidos por meio da sondagem periodontal, como a padronização da sonda periodontal, levando a uma incorreta mensuração clínica ${ }^{11}$. Por isso, este estudo teve como propósito avaliar a padronização de diferentes marcas de sondas periodontais milimetradas a fim de verificar a variabilidade e reprodutibilidade das mesmas.

A OMS estabelece alguns parâmetros dimensionais para que a sonda periodontal seja considerada ideal, a fim de se evitar erros no momento da sondagem, no entanto, tais parâmetros só podem ser empregados para as sondas do tipo OMS, não podendo ser aplicados aos demais tipos de sondas periodontais. Entretanto, estudos realizados a fim de se estabelecer a sonda ideal acabam por se basear nos parâmetros estabelecidos pela OMS, pela inexistência de parâmetros específicos à cada tipo de sonda ${ }^{3,10,17}$. Contudo, os resultados obtidos no presente estudo demonstram discrepâncias entre os parâmetros analisados para todas as marcas analisadas.

Apesar do peso da sonda recomendado pela OMS ser de $4,5 \mathrm{~g}$, todas as sondas analisadas neste estudo, de todas as marcas, inclusive as sondas do tipo OMS, apresentaram valores muito acima do recomendado. Estudos semelhantes também encontraram valores acima do preconizado, apesar destes estudos terem avaliado somente algumas marcas de todas as avaliadas neste ${ }^{10}$. $O$ propósito de 0 peso da sonda ser de 4,5g tem como finalidade que o peso total da sondagem, somado à força aplicada pelo examinador, seja de 20 a $25 \mathrm{~g}$, influenciando negativamente nos valores de profundidade e sangramento à sondagem ${ }^{10,11,18}$. $\mathrm{O}$ diâmetro do cabo também acaba por interferir no peso das sondas. No presente estudo, todas as sondas avaliadas apresentaram a medida do diâmetro do cabo muito além do estabelecido pela OMS. Para as sondas do tipo OMS, a da marca Trinity apresentou a medida mais aproximada da preconizada pela OMS $(3,95 \mathrm{~mm})$ e a do tipo Williams, da marca Fava $(4,96 \mathrm{~mm})$.

O diâmetro da esfera da ponta ativa, de acordo com os parâmetros da OMS, deve ser de $0,5 \mathrm{~mm}$. No entanto, no presente estudo, para as sondas do tipo OMS avaliadas, somente a da marca Hu-Friedy seguiu essa especificação $(0,51 \mathrm{~mm})$, as demais marcas apresentaram valores acima do recomendado. Medida semelhante fora encontrada no estudo desenvolvido por Van der Zee ${ }^{5}$ et al. para a sonda da marca Hu-Friedy. Como dito anteriormente, não há recomendações para o diâmetro da ponta ativa para as sondas do tipo Williams. Porém, segundo Magalhães ${ }^{18}$ et al., esses valores podem variar entre $0,35 \mathrm{~mm}$ a $0,63 \mathrm{~mm}$. Para as marcas avaliadas no presente estudo, somente a sonda tipo Williams da Fava apresentou medida do diâmetro da ponta ativa superior à $0,63 \mathrm{~mm}$. Van der $\mathrm{Zee}^{5}$ et al. afirmam que sondas periodontais com pontas ativas muito finas tendem a superestimar a profundidade de sondagem em tecidos inflamados e sondas espessas subestimariam a profundidade de sondagem em condições de restabelecimento de saúde periodontal.

As marcações milimetradas avaliadas neste estudo para as sondas do tipo Williams foram de $0-1 \mathrm{~mm}, 0-5 \mathrm{~mm}, 0-7 \mathrm{~mm}$ e $0-10 \mathrm{~mm}$, sendo a da marca Hu-Friedy a que apresentou as medidas mais precisas. Corroborando nossos resultados, César ${ }^{7}$ et al., utilizando um analisador de imagens e um paquímetro digital, também concluiu que a sonda da marca HuFriedy apresentava medidas mais precisas, aproximando-se muito das medidas padrão. Assim como no presente estudo, Winter ${ }^{17}$ concluiu que a maioria das sondas não apresenta precisão em suas medidas. Neste último, as medidas mais precisas encontradas foram nas sondas da marca Hu-Friedy, bem como nesta pesquisa. As marcações milimetradas avaliadas nas sondas do tipo OMS foram $0-3,5 \mathrm{~mm}, 0-5,5 \mathrm{~mm}, 0-8,5 \mathrm{~mm}$ e $0-11,5 \mathrm{~mm}$ e, assim como na sonda do tipo Williams, a sonda da Hu-Friedy foi a marca que apresentou medidas mais precisas. As demais marcas tiveram medidas muito semelhantes, no entanto a da marca Fava foi a que apresentou medidas muito aquém dos valores estipulados pela OMS. Porém, em um estudo desenvolvido por Neto ${ }^{19}$, avaliando sondas OMS, as da marca Quinelato foram as que tiveram melhores resultados e, piores a da marca Golgran. Esta diferença entre nossos resultados pode ser devido ao fato de que neste último estudo, a sonda da marca $\mathrm{Hu}$ Friedy não tenha sido analisada.

Se levarmos em consideração o custo, a sondagem manual ainda é o procedimento de eleição quando comparada à sondagem digital. Para ambos os tipos de sonda (OMS e Williams), as da marca Hu-Friedy foram as que apresentaram maiores valores. Talvez, isso se deva ao fato de que esta marca fora a que apresentou melhores resultados quanto às marcações milimetradas. 
CONCLUSÃO

Perante os resultados obtidos e respeitando o limite da metodologia, concluiu-se que as seis marcas comerciais avaliadas, tanto das sondas do tipo OMS quanto do tipo Williams, apresentaram algum tipo de alteração dimensional e, portanto, nenhuma sonda possui exatamente todas as características ideais recomendadas pela OMS. Porém, as sondas da marca Hu-Friedy, tanto do tipo OMS quanto Williams, foram as que apresentaram melhores marcações milimetradas de todas as marcas avaliadas.

\section{AGRADECIMENTOS}

Os autores agradecem às marcas Fava, Quinelato e Trinity pelo envio das sondas para que este estudo pudesse ser realizado.

\section{REFERÊNCIAS}

1. Medlicott NJ, Rathbone MJ, Tucker IG, Holborow DW. Delivery systems for the administration of drugs to the periodontal pocket. Adv Drug Deliv Rev.1994;13(1/2):181-203.

2. Novak MJ. Classificação das Doenças e Condições que a fetam o Periodonto. In: Newman MG, Takei HH, Carranza FA. Periodontia Clínica. 9. ed. Rio de Janeiro: Guanabara Koogan; 2004. p.58-65.

3. Tahin CM, Barbosa CS, Mota OML, Pereira SLS, Lima DLF, Carlos MX. Avaliação da Padronização de Sondas Periodontais Tipo Williams. R Periodontia. 2007; 17(3):86-9.

4. Lindhe J, Karring T, Lang NP. Tratado de periodontia clínica e implantologia oral. 8. ed. Rio de Janeiro: Guanabara Koogan; 2005.

5. Van der Zee E, Davies EH, Newman HN. Marking width, calibration from tip and tine diameter of periodontal probes. $\mathrm{J}$ Clin Periodontol. 1991;18(7):516-20.

6. Carranza FA, Newman MG. Periodontia clínica. 8.ed. Rio de Janeiro: Guanabara Koogan; 1997.

7. César JB, Nogueira GR, Casati MZ, Machado MA, Tramontina VA, Nociti Jr FHN. Avaliação da padronização de sondas periodontais: marcas milimetradas e diâmetro das pontas. Maio-Dezembro 1999 [ cited 2005 Aug18] Available from: URL: http:// www.oraltech.com.br/biblioteca/hu001

8. Rocha EF. Variabilidade na profundidade clínica de sondagem utilizando sonda convencional e de pressão controlada. Salusvita. 2003;22(22):209-17.

9. Pontes IV, Machado JPP, Luna de Almeida $\mathrm{R}$, Mendonça JS, Pereira SLS. Análise do controle de placa em alunos de graduação em odontologia. R Periodontia. 2007;17(3): 105-9.

10. Penteado IAM, Albuquerque AC, Medeiros CWO, Oliveira DP, Santos NB. Alterações dimensionais das sondas periodontais do tipo Williams submetidas a 100 ciclos de autoclavação. R Periodontia. 2010;2:75-81.

11. Costa RCC, Koser LR, Alves PM. Sondas periodontais convencionais: uma revisão. Rev ABO Nac. 2007;15(5):296-99.

12. Camargo GACG, Abreu MGLA, Cordeiro RS, Crespo MA, Wenderoscky LF. Aspectos clínicos, microbiológicos e tratamento periodontal em pacientes fumantes portadores da doença periodontal crônica: revisão de literatura. Rev bras odontol. 2016;73(4):325-30.

13. Duarte CA, Castro MVM. Cirurgia Periodontal Pré-protética, Estética e Peri-implantar. 4. ed. São Paulo: Grupo Gen/Santos; 2015.

14. Research, Science and Therapy Committee. Position Paper: Diagnosis of Periodontal Diseases. J Periodontol. 2003;74(8):1237-247.

15. Armitage GC. The complete periodontal examination. Periodontol 2000. 2004;34: 22-33.

16. Moreira $\mathrm{CHC}$, Fernandes $\mathrm{T}$, Antoniazzi $\mathrm{R}$, Rosing CK. Recursos diagnósticos de doenças periodontais utilizados por cirurgiões-dentistas de três cidades do Rio Grande do Sul. Rev Soc Bras Periodontol. 2007;17(1):36-40.

17. Winter AA. Measurement of the millimeter markings of periodontal probes. J Periodontol. 1979;50(9):483-85.

18. Magalhães D, Compoli MAO, Teixeira CPR. Avaliação da precisão de sondagem periodontal. Rev Odontol Bras Central 1993; 3(7):4-6.

19. Andrade Neto JM, Martins EOB. Avaliação da milimetragem de sondas periodontais de uma instituição de ensino superior de Aracaju/SE (UNIT-SE). Aracaju: Universidade de Tiradentes; 2014. 


\section{CONFLITO DE INTERESSES}

Os autores declaram não haver conflitos de interesse

\section{AUTOR PARA CORRESPONDÊNCIA}

\section{Juliane Pereira Butze}

Curso de Odontologia,

Centro Universitário da Serra Gaúcha - FSG

Rua Os Dezoito do Forte, 2366. Bairro São Pelegrino

95020-472 Caxias do Sul - RS, Brasil

E-mail: juliane.butze@fsg.edu.br 